\title{
Prognosis of Severe and Critical Patients Who Have Recovered from COVID-19: A Three-Month Follow-Up
}

\author{
Chang-Hao Zhong ${ }^{a}$ Zi-Qing Zhou ${ }^{a}$ Feng Ye ${ }^{a}$ Wen-Liang Guo ${ }^{a}$ Wei-Li Gu ${ }^{a}$ \\ Zu-Yuan Guo ${ }^{a}$ Shi-Yue Li ${ }^{a}$ \\ a State Key Laboratory of Respiratory Diseases, National Clinical Research Center for Respiratory Diseases, \\ Guangzhou Institute of Respiratory Health, The First Affiliated Hospital of Guangzhou Medical University, \\ Guangzhou, China
}

\section{Dear Editor,}

COVID-19 has been prevalent for more than 8 months, and as many as 10 million patients have recovered from COVID-19. However, the possible long-term outcomes with regard to physical, psychological, and social aspects in patients who recovered from COVID-19 are not yet fully understood. It has been observed that COVID19-recovered patients can develop impairment in multiorgan systems [1]. Also, most patients who recover from COVID-reportedly experience mental and social stress due to the infection and being stigmatized by society. Given this situation, regular follow-up of recovered patients, particularly severe and critical patients who required intensive care unit (ICU) admission, may be necessary to determine whether they have any other physical and psychological impairments after discharge. However, studies on the clinical outcomes of recovered patients are still lacking. Hospitals and doctors may face difficulties offering regular follow-up checks to recovered patients due to the uncertainty of infectiousness and insufficient public medical resources. Mobile devices are a low-cost source of technology for addressing healthcare needs, which can be used to exchange health information through connected apps or calls [2]. Since more than 1 billion people in
China use the WeChat app as a communication tool, remote network follow-up through WeChat app may be of great use to COVID-19-recovered patients due to the rapid, simple, and economically feasible nature of the communication between patients and doctors. The aim of this work is to investigate the prognosis of severe and critical patients who have been discharged from the hospital by collecting data of their general physical and psychological condition through a WeChat-based survey system.

Confirmed severe and critical cases of COVID-19 who required ICU admission and were discharged from the isolation ward of The First Affiliated Hospital of Guangzhou Medical University from March 1 to March 30 were enrolled into this study. The diagnostic criteria were according to interim guide for novel coronavirus pneumonia published by the National Health Commission of the People's Republic of China [3, 4].

A mini-program (Levsin; Sightfuture Medical Technology, China) was developed and implanted in the WeChat app, which included a patient side module, a doctor side module, and a backstage administration module

Drs. Chang-Hao Zhong and Zi-Qing Zhou are co-first authors. 




Fig. 1. Working pattern of the WeChat app-based follow-up program. A mini-program which included a patient side module, a doctor side module, and the BAM were implanted in the WeChat app. The BAM could push questionnaires to patients and send warnings to doctors. Doctors could collect data from BAM and send medical guidance to the patients.

(BAM). The BAM pushed the questionnaires to the patient side automatically and collected the data of the survey. Warnings could be sent to doctors if patients reported a significant abnormality in their daily survey, and doctors could send medical guidance to patients through the doctor side module. Figure 1 shows the pattern of the follow-up program. An infrared electric clinical thermometer (YHW-2; Yuwell, China) and a hand-held pulse oximeter (YX303; Yuwell) were sent to the patients to monitor their vital signs. The following information was collected through the WeChat-based survey: (1) patents' vital signs, including body temperature, heart rate, and blood oxygen saturation; (2) postdischarge sequela; (3) self-rating scales, including the Self-Rating Anxiety Scale (SAS) and the SelfRating Depression Scale (SDS), to evaluate psychological disorders; (4) health-related quality of life (HRQoL), accessed by the Chinese version of the SF-36 survey (MOS SF-36, which includes 8 multiple-item domains that assess physical functioning, social functioning, role limitation due to physical problems, role limitation due to emotional problems, mental health, bodily pain, vitality, and general health) [5] and the Modified Medical Research Council (mMRC) dyspnea scale. Patients' vital signs and postdischarge sequela were collected every day. Self-rating questionnaires were collected every month.

Three-Month Follow-Up of COVID-19Recovered Patients
A total of 9 consecutive discharged patients with a mean age of 48.6 years were recruited, including 3 cases of severe pneumonia and 6 cases of critical illness. Three of the 9 patients had at least 1 coexisting illness (i.e., 1 had diabetes and coronary disease, 1 had hypertension, and 1 had hypertension and diabetes). The mean duration of ICU stay was $40.2 \pm 11.2$ days and the mean time from onset of the disease to discharge was $62.7 \pm 16.3$ days in severe cases; 5 of the 9 patients reported 1 or more sequelae when they were discharged, which included sensation of palpitations $(n=1)$, fatigue $(n=2)$, dizziness $(n=$ $2)$, coughing $(n=1)$, chest pain $(n=1)$, insomnia $(n=1)$, and tinnitus $(n=1)$. The patients' baseline characteristics are shown in Table 1.

Eight of the 9 patients finished the 3-month postdischarge WeChat follow-up. One patent discontinued follow-up on the second day due to incompatibility of the WeChat push notification with his phone, and a telephone call follow-up was done instead. All 9 patients had stable vital signs within the normal range during the 3-month postdischarge follow-up period, with a mean heart rate of $88 \pm 12$ beats/min, a mean body temperature of $36.5 \pm 0.3^{\circ} \mathrm{C}$, and a mean $\mathrm{SpO}_{2}$ level of $98.2 \pm 0.2 \%$ with room air at rest. Chest CT follow-ups were conducted 2 weeks after discharge and showed multiple patchy ground glass opacities that appeared to shrink and be less dense in 8 patients, and parenchymal fibrosis changes occurred in 1 patient (No. 1) compared to the initial CT imaging. He also reported a low-grade fever within a range of 37.3$37.5^{\circ} \mathrm{C}$, which lasted for a month. However, a repeated nucleic test and a chest CT did not show evidence of disease recurrence. Patient No. 9 reported new-onset chest pain at 1 month and was asked to return to the hospital. Acute myocardial infarction was excluded by electrocardiogram and a myocardial enzyme test in this patient. All of the 5 patients who had postdischarge manifestations sequentially recovered within the 3 -month follow-up. Patient No. 5 had a positive RT-PCR test on the 19th day postdischarge, but there were no changes in the clinical examination and or chest CT findings compared to previous results. Moreover, the patient did not report contact with any person with respiratory symptoms. There was a significant impairment in the HRQoL and psychological state 3 months after discharge among the 9 patients, and tough SF-36, SAS, and SDS self-reported scale score values appeared at 1 month. Impairments of role limitation due to physical problems, general health, social functioning, emotional problems, and vitality were more obvious, that the mean domain scores were lower than 60 within 3 months. Eight patients had grade 1 on the mMRC scale 
Table 1. Patient characteristics

\begin{tabular}{|c|c|c|c|c|c|c|c|c|c|}
\hline Patient No. & 1 & 2 & 3 & 4 & 5 & 6 & 7 & 8 & 9 \\
\hline Age, years & 49 & 53 & 50 & 41 & 26 & 67 & 42 & 50 & 59 \\
\hline Sex & Male & Male & Male & Male & Male & Male & Male & Male & Male \\
\hline Severity & Critical & Critical & Severe & Critical & Severe & Critical & Severe & Critical & Critical \\
\hline Coexisting illness & None & $\begin{array}{l}\text { Diabetes } \\
\text { and coronary } \\
\text { disease }\end{array}$ & None & $\begin{array}{l}\text { Hyper- } \\
\text { tension }\end{array}$ & None & None & None & $\begin{array}{l}\text { Hypertension } \\
\text { and diabetes }\end{array}$ & None \\
\hline Duration of ICU stay, days & 41 & 39 & 39 & 25 & 59 & 46 & 22 & 46 & 45 \\
\hline Total hospital stay, days & 56 & 68 & 68 & 40 & 89 & 61 & 41 & 81 & 61 \\
\hline Sequelae at discharge & Fever & None & None & None & Anxiety & $\begin{array}{l}\text { Fatigue, } \\
\text { palpitations, } \\
\text { and insomnia }\end{array}$ & None & Coughing & $\begin{array}{l}\text { Dizziness, } \\
\text { tinnitus, and } \\
\text { chest pain }\end{array}$ \\
\hline
\end{tabular}

on the day of discharge and showed a trend toward improvement over time.

Studies with respect to the long-term outcomes of recovered patients are still lacking. In this study, we reveal the 3-month outcomes and the prognosis of severe and critical patients with regard to psychological and physical aspects. Postdischarge CT scanning showed that consolidation was gradually absorbed in the majority of patients, evidenced by a decreased crazy-paving pattern and ground glass opacities. It has also been reported that in patients with COVID-19 pulmonary fibrosis may develop in the second week after symptom onset [6]. In our study, 1 of the patients presented with CT evidence of fibrotic changes, consistent with the CT findings in SARS, which raises the concern that some patients with COVID-19 may have the potential to develop lung fibrosis and lung function impairment [7]. The physical state was stable in patients who recovered from severe and critical illness, evidenced by vital signs within the normal range during the 3-month follow-up. Recent studies have revealed that COVID-19 manifests as infections in multiorgan systems, inflicting damage to the heart, the brain, the eyes, the kidneys, the digestive tract, and the lungs [8-10]. Patients in this study reported having intrapulmonary and extrapulmonary manifestations after discharge, which include sensations of chest pain, palpitations, fatigue, dizziness, coughing, and tinnitus. However, all of the manifestations were relatively mild and gradually relieved. Indicated a transient infectious with good recovery in multi-organ system in these patients. All of the COVID-19-recovered patients showed a significantly impaired HRQoL and psychophysical state for a longer time, evidenced by a reduced SF-36 scale score and raised SAS and SDS scale scores in the 3-month followup. Previous studies on ALI/ARDS related to SARS or other disease have reported the impairment of HRQoL domains; especially physical functioning, physical problems, general health, and social functioning were correlated with impaired lung function [7]. The results of the present study coupled with the published evidence suggest that lung function impairment may also occur in patients who have recovered from COVID-19. In the present study, follow-up data was collected through WeChat. By establishing this telephone app-based follow-up system, doctors and researchers could monitor patients' health status through patients' daily report of vital signs and self-reported scales in a convenient way. Furthermore, medical guidance from doctors could be provided through the system when patients present with significant abnormalities in vital signs and self-reported scales, which helped to lower medical costs, reducing the risk of transmission and further improving patients' compliance.

There are limitations to our study. Firstly, the patients in this study did not attend an out-patient follow-up in 3 months to confirm their health state (e.g., undergo CT, PEF examinations, and nucleic acid tests). In a telephonebased remote follow-up, vital signs and HRQoL from selfreported scales could reflect patients' health state well. Secondly, the lack of baseline SAS, SDS, and SF-36 scores prior to the illness makes it difficult to evaluate the changes in these self-reported scale scored induced by COVID-19 infection. None of the patients had mental disorders or chronic respiratory diseases, so it should be ac- 
ceptable to assume that the self-reported scales of the patients would be normal. Finally, we recognize that the small sample size limits the generalizability of our findings, and multicenter long-tern follow-up studies should be conducted to understand the possible outcomes of COVID-19-recovered patients.

In conclusion, COVID-19 patients with severe and critical illness may recover from the disease but be left with mutiorgan manifestations and psychological disorders. Telephone app-based remote follow-up may be of great use in the management of COVID-19-recovered patients by establishing simple and effective communication between patients and doctors.

\section{Acknowledgement}

We thank Sightfuture Medical Technology (Shanghai) Co., Ltd., who supported us with the technical operation of the WeChat follow-up system.

\section{Statement of Ethics}

All of the patients were constantly informed about this study and the study procedures were approved by the ethics committee of The First Affiliated Hospital of Guangzhou Medical University.

\section{Conflict of Interest Statement}

All of the other authors declare no potential conflict of interests.

\section{Funding Sources}

Dr. Zhong declares that he received a Special Fund for Science and Innovation Strategies of Guangdong Province from the Zhongnanshan Medical Foundation of Guangdong Province (2020B1111340020). None of the funding sources had any role on the study.

\section{Author Contributions}

C.-H. Zhong, Z.-Q. Zhou, F. Ye, W.-L. Guo, W.-L. Gu, and Z.Y. Guo performed the literature search and drafted this paper. C.H. Zhong, Z.Q. Zhou, W.-L. Guo, Z.-Y. Guo, and W.-L. Gu were responsible for the patient follow-up. C.-H. Zhong, Z.-Q. Zhou, W.-L. Guo, Z.-Y. Guo, and W.-L. Gu contributed to the data collection, analysis, and interpretation; C.-H. Zhong and Z.-Q. Zhou performed statistical analyses. C.-H. Zhong, Z.-Q. Zhou, F. Ye, and S.-Y. Li contributed to the study conception; S.-Y. Li critically revised this paper and approved the final version for submission.

\section{References}

1 Balachandar V, Mahalaxmi I, Subramaniam M, Kaavya J, Senthil Kumar N, Laldinmawii $\mathrm{G}$, et al. Follow-up studies in COVID-19 recovered patients - is it mandatory? Sci Total Environ. 2020 Aug;729:139021.

2 Pillay Y, Motsoaledi PA. Digital health in South Africa: innovating to improve health. BMJ Glob Health. 2018 Apr;3 Suppl 2:e000722.

3 World Health Organization. Clinical management of severe acute respiratory infection when COVID-19 is suspected. [cited 202013 March ]. Available from: https://www.who. int/publications-detail/clinical-management-of-severe-acute-respiratoryinfectionwhen-novel-coronavirus-(ncov)-infectionis-suspected
4 National Health Commission \& State Administration of Traditional Chinese Medicine. Diagnosis and Treatment Protocol for Novel Coronavirus Pneumonia (Trial Version 7). [cited 2020 March 3]. Available from: https:// www.who.int/docs/default-source/wpro--documents/countries/china/covid-19-briefing-nhc/1-clinical-protocols-for-the-diagnosis-and-treatment-of-covid-19-v7. pdf?sfvrsn $=$ c6cbfba4_2

5 Lam CL, Gandek B, Ren XS, Chan MS. Tests of scaling assumptions and construct validity of the Chinese (HK) version of the SF-36 Health Survey. J Clin Epidemiol. 1998 Nov; 51(11):1139-47.

6 Zheng Z, Yao Z, Wu K, Zheng J. Patient follow-up after discharge after COVID-19 pneumonia: considerations for infectious control. J Med Virol. 2020 May.
7 Hui DS, Joynt GM, Wong KT, Gomersall CD, Li TS, Antonio G, et al. Impact of severe acute respiratory syndrome (SARS) on pulmonary function, functional capacity and quality of life in a cohort of survivors. Thorax. 2005 May;60(5):401-9.

8 Mao L, Jin H, Wang M, Hu Y, Chen S, He Q, et al. Neurologic Manifestations of Hospitalized Patients With Coronavirus Disease 2019 in Wuhan, China. JAMA Neurol. 2020 Jun; 77(6):683-90.

9 Sommer A. Humans, Viruses, and the Eye-An Early Report From the COVID-19 Front Line. JAMA Ophthalmol. 2020 May;138(5):578-9.

10 Xiong TY, Redwood S, Prendergast B, Chen $M$. Coronaviruses and the cardiovascular system: acute and long-term implications. Eur Heart J. 2020 May;41(19):1798-800. 